\title{
Deflating infinite Coxeter groups to finite groups
}

\author{
Christopher S. Simons
}

\section{Introduction}

In $[\mathbf{5}]$ it is shown, using the Ivanov-Norton theorem $[\mathbf{8 , 1 1}]$, that the Bimonster, MI 2, is presented by the Coxeter relations of the 26 node incidence graph of the projective plane of order 3 along with the additional relations that all free 12 -gons of this diagram generate symmetric groups $S_{12}$. The purpose of this paper is to consider an easier version of this presentation by replacing the 26 node incidence graph of the projective plane of order $3, \operatorname{Inc}\left(\mathbb{P}_{3}\right)$, by the 14 node incidence graph of the projective plane of order $2, \operatorname{Inc}\left(\mathbb{P}_{2}\right)$. The maximum free $n$-gons of $\operatorname{Inc}\left(\mathbb{P}_{2}\right)$ are 8 -gons so the additional non-Coxeter relations are that all free 8 -gons generate symmetric groups $S_{8}$. We prove that the resulting group is $O_{8}^{-}(2): 2$ (in the Atlas [2] notation).

This new presentation is in some sense more satisfying than the related Monstrous presentation as it is possible to understand it in an elementary and self contained fashion. We hope to use it and other similar presentations to explain the finite simple Monster group in a nonsporadic manner. That $O_{8}^{-}(2): 2$ acts as a pseudomonster is not entirely new as it is the unique nonsymmetric $S(3,8)$ group while the $\mathbb{M} 22$ is the unique nonsymmetric $S(5,12)$ group [1].

To avoid unnecessary complications, in Section 2 we restrict our attention to the $O_{8}^{-}(2): 2$ case. Comments on the Bimonster and general cases are left to Section 3. A useful basic reference on Coxeter and reflection groups is [7]. We also provide some relevant references for the motivating Monstrous case.

\section{Results}

The projective plane of order $2, \mathbb{P}_{2}$, consists of 7 points and 7 lines as shown in Figure 1. Each line consist of 3 points, any two lines uniquely determine a point and there is a duality between points and lines. The incidence graph of the projective plane of order 2 , Inc $\left(\mathbb{P}_{2}\right)$, therefore has 14 nodes (one for each of the 7 points and 7 lines of $\mathbb{P}_{2}$ ). Two nodes are joined exactly when one is a point, one is a line and the point lies on the line. The graph $\operatorname{Inc}\left(\mathbb{P}_{2}\right)$ has valence 3 and is shown in Figure 2. The indices $i$ range over $\{1,2,3\}$ so that some of the vertices of the figure correspond to 3 nodes of $\operatorname{Inc}\left(\mathbb{P}_{2}\right)$. Single lines in the figure indicate that two of these nodes are joined just if they share the same index. Double lines indicate that two of these nodes are joined just if their indices differ.

1991 Mathematics Subject Classification. Primary 20D06, 20D08, 20F55.

The author was supported in part by an NSERC postdoctoral fellowship. 


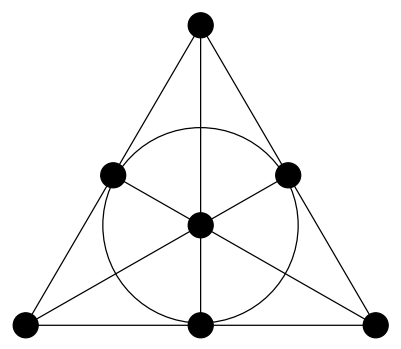

Figure $1 . \mathbb{P}_{2}$

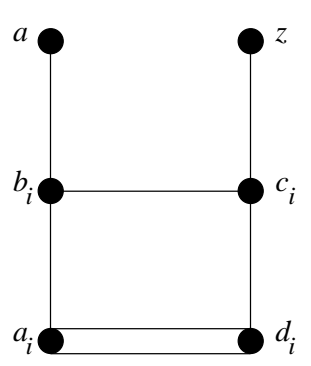

Figure 2. $\operatorname{Inc}\left(\mathbb{P}_{2}\right)$

To $\operatorname{Inc}\left(\mathbb{P}_{2}\right)$ or any graph $\Gamma$ we can associate a Coxeter group $H$ generated by the nodes and subject to the graph's Coxeter relations. The Coxeter relations are that all nodes are involutions $\left(\alpha^{2}=1\right)$, that any two unjoined nodes commute $\left.\left((\alpha \beta)^{2}=1\right)\right)$ and that the product of any two joined nodes has order $\left.3\left((\alpha \beta)^{3}=1\right)\right)$.

It is important for us that all Coxeter groups are isomorphic to reflection groups. This allows us to work geometrically. Each generating involution corresponds to the reflection, $x \longmapsto x-(x, \alpha) \alpha$, in a root $\alpha$ (we use the same name for both the root and the associated reflection). The inner products of these roots are such that $(\alpha, \alpha)=2$ for all roots $\alpha,(\alpha, \beta)=0$ for $\alpha, \beta$ unjoined and $(\alpha, \beta)=-1$ for $\alpha, \beta$ joined.

The $\operatorname{Inc}\left(\mathbb{P}_{2}\right)$ Coxeter group is infinite. In order to present the finite group $O_{8}^{-}(2): 2$ we must adjoin some additional relations. We make use of the following convention introduced in [5].

Definition 2.1. Let $\mathbb{Z}^{m}: G$ be a group (usually an affine Coxeter group). To deflate this group is to impose the relations that make the translations $\mathbb{Z}^{m}$ trivial. Similarly to biflate, triflate or $k$-flate $\mathbb{Z}^{m}: G$ is to make the translations have order 2,3 or $k$ respectively with the result that $\mathbb{Z}^{m}$ becomes $2^{m}, 3^{m}$ or $k^{m}$. Usually we view $\mathbb{Z}^{m}: G$ as a subgroup of a group $H$. The new relations are then imposed on $H$.

$\mathbb{Z}^{m}$ is the free Abelian group of rank $m$. The group notation $A: B$ is used for a semidirect product of $A$ and $B$. Note that $A$ will be a normal subgroup of $A: B$. Below we use lower case letters for spherical Dynkin diagrams and upper case letters to the associated affine Dynkin diagrams.

We deflate affine $A_{7}$ Coxeter groups $\mathbb{Z}^{7}: S_{8}$. Since the affine $A_{7}$ diagram is just a free 8 -gon (a graph with 8 nodes joined precise as the vertices of an octagon), we say that we are deflating a free 8 -gon. For example, if $\alpha_{0}, \ldots, \alpha_{7}$ are the involutions 


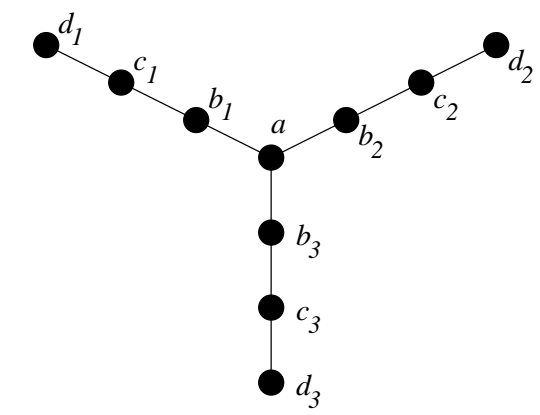

Figure 3. $Y_{333}$ diagram

of an 8-gon then we can deflate this 8-gon by adding the relation $\alpha_{0}=\alpha_{1}^{\alpha_{2} \cdots \alpha_{7}}$. This should be clear in a moment.

We now state the main result of this paper.

Theorem 2.2. If we deflate all free 8-gons of the Inc $\left(\mathbb{P}_{2}\right)$ Coxeter group we obtain the finite group $O_{8}^{-}(2): 2$.

In general it is very difficult to identify a group from its presentation. To prove Theorem 2.2 we therefore take an indirect approach.

We start with a (small) graph $\Gamma_{0}$ : the $Y_{333}$ digram (Figure 3 ). We consider a group $G$ satisfying its Coxeter relations. We then extend $\Gamma_{0}$ by repeatedly adjoining affine $A_{7}$ extending nodes. The closure of $\Gamma_{0}$ under this extension is denoted $\Gamma$. Of course $G$ satisfies the Coxeter relations of $\Gamma$.

Adjoining $A_{7}$ extending nodes is straightforward. Let the nodes $\alpha_{1}, \ldots, \alpha_{7}$ form an $a_{7}$ subdiagram ( 7 nodes forming a chain). Its Coxeter group is $S_{8}$ and the nodes can be viewed as the transpositions $(0,1), \ldots,(6,7)$ of $S_{8}$. The extending node $\alpha_{0}$ is then the transposition $(7,0)$ and can be written as $\alpha_{1}^{\alpha_{2} \cdots \alpha_{7}}$. In order to make use of $\alpha_{0}$ in further $A_{7}$ extensions we must determine its Coxeter relations with other nodes of $\Gamma$. We do this by treating $G$ as (the quotient of) a reflection group. We carefully choose root vectors $\alpha_{1}, \ldots, \alpha_{7}$. These must have norm 2, inner product 0 when unjoined and inner product -1 when joined. (Ensuring inner product -1 requires some care since while the sign of a root does not effect the corresponding reflection element it is important here. The choice of signs does not have to be consistent between different $a_{7}$ subdiagrams.) We then have by Coxeter theory that the extending root $\alpha_{0}$ is $-\left(\alpha_{1}+\cdots+\alpha_{7}\right)$. Inner products can determine the Coxeter relations between the new node $\alpha_{0}$ and other nodes $\alpha$ of $\Gamma$. If $\left(\alpha_{0}, \alpha\right)=0$ then $\alpha_{0}$ and $\alpha$ are unjoined. If $\left(\alpha_{0}, \alpha\right)= \pm 1$ then $\alpha_{0}$ and $\alpha$ are joined.

Two roots corresponding to the same element of $G$ are said to be equivalent and they share the same node of $\Gamma$.

We prove that if $\Gamma$ (for $\Gamma_{0}$ still the $Y_{333}$ diagram) has no more than 14 distinct nodes then $G$ is $O_{8}^{-}(2): 2$. Upon examination of the relations used in this proof we then get Theorem 2.2 .

Theorem 2.3 (14 implies $\left.O_{8}^{-}(2): 2\right)$. Let $G$ be a group (of order greater than 2) generated by and satisfying the Coxeter relations of the $Y_{333}$ diagram. If after closure of the generating $Y_{333}$ diagram by adjoining $A_{7}$ extending nodes there are no more than 14 nodes, then $G$ is $O_{8}^{-}(2): 2$. 


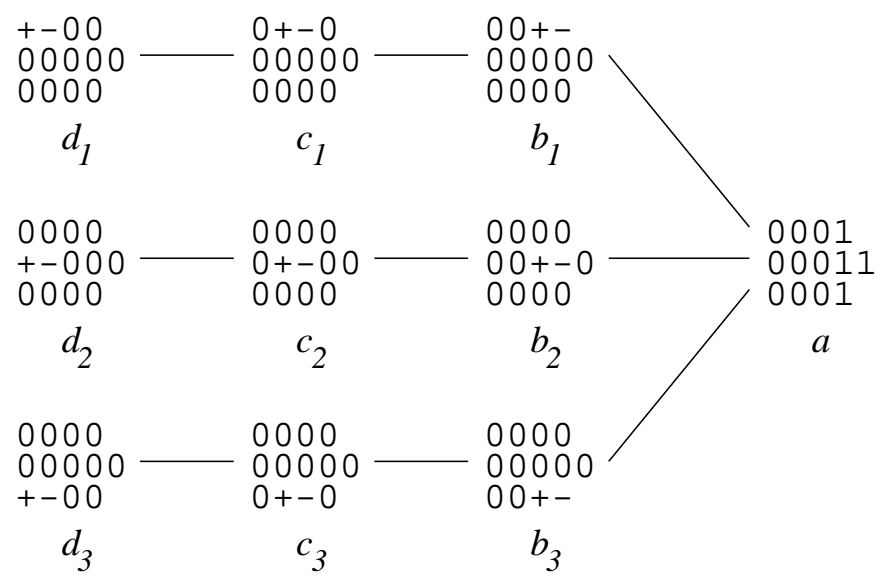

Figure 4. The fundamental roots of $Y_{333}$

Insisting that there are no more than 14 nodes imposes some non-Coxeter relations, the equivalence of certain roots, on the $Y_{333}$ infinite Coxeter group with the result that we get a presentation for $O_{8}^{-}(2): 2$. In fact we show that $\Gamma$ has exactly 14 nodes and is $\operatorname{Inc}\left(\mathbb{P}_{2}\right)$. We do assume for now that $G$ is not trivial (of order 1 or 2). This is justified since by its construction the group $O_{8}^{-}(2): 2$ will satisfy the conditions.

We start with $\Gamma_{0}$ being the $Y_{333}$ diagram and close under $A_{7}$ extension. In order to proceed we use the following coordinate system. Often we will use + to denote 1 and - to denote -1 .

We have a space of 13 coordinates

$$
\begin{array}{ccccc}
a & b & c & d & \\
e & f & g & h & t \\
i & j & k & l &
\end{array} \text { with quadratic form } a^{2}+\cdots+l^{2}-t^{2} .
$$

In this system the 10 original roots of $Y_{333}$ are as indicated in Figure 4.

All the vectors satisfy the following relations:

$$
\begin{gathered}
a+b+c+d=t \\
e+f+g+h=t \\
i+j+k+l=t .
\end{gathered}
$$

Thus $t$ is redundant, so can be omitted.

We now extend the $Y_{333}$ diagram by adjoining $A_{7}$ extending roots to obtain $\Gamma$. We do this by finding free $a_{7}$ subdiagrams and adding the $A_{7}$ extending nodes. We stress that in all cases used the $a_{7}$ roots satisfy the standard inner product conditions. The choice of signs of the $a_{7}$ roots is important. If $\alpha$ is a root then we use $\underline{\alpha}$ to denote the negative of $\alpha$. The signed sum of the $a_{7}$ roots is the $A_{7}$ extending root.

The $Y_{333}$ diagram has 10 nodes. 


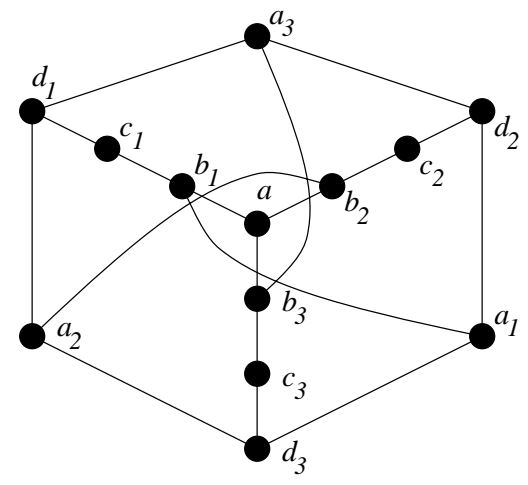

FiguRE 5. $a_{1}, a_{2}, a_{3}$

We use the subdiagram $d_{1}-c_{1}-b_{1}-a-b_{2}-c_{2}-d_{2}$ to get

$$
a_{3}=\begin{array}{lllll}
1 & 0 & 0 & 0 & \\
1 & 0 & 0 & 0 & 1 \\
0 & 0 & 0 & 1 &
\end{array} .
$$

Checking inner products we see that $a_{3}$ is joined to $b_{3}$ in addition to $d_{1}$ and $d_{2}$. It is unjoined with the other nodes of $\Gamma$.

By the obvious $S_{3}$ symmetry of the the $Y_{333}$ diagram we similarly obtain $a_{1}$ and $a_{2}$.

So far we have 13 roots as shown in Figure 5. By checking inner products with the original 10 roots of $Y_{333}$ we find that the roots are distinct. So under the conditions of Theorem 2.3 there is only one more node of $\Gamma$ still to be found.

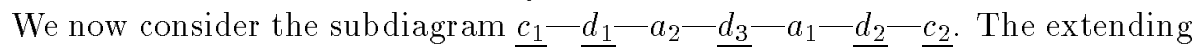
root is

$$
z_{3}=\begin{array}{lllll}
0 & 0 & 1 & 1 & \\
0 & 0 & 1 & 1 & 2 \\
1 & 1 & 0 & 0 &
\end{array} .
$$

Checking inner products we see that $z_{3}$ is joined to $c_{1}, c_{2}, c_{3}$ and is unjoined with the other nodes of $\Gamma$.

We can similarly obtain $z_{1}$ and $z_{2}$. These have the same Coxeter relations as $z_{3}$. So by the conditions of Theorem 2.3 we have that the $z_{i}$ are equivalent, $z_{1} \equiv z_{2} \equiv z_{3}$ :

$$
\begin{aligned}
& \begin{array}{llllllllllll}
1 & 1 & 0 & 0 & 0 & 0 & 1 & 1 & 0 & 0 & 1 & 1
\end{array} \\
& \begin{array}{lllllllllllllllll}
0 & 0 & 1 & 1 & 2 & \equiv & 1 & 1 & 0 & 0 & 2 & 0 & 0 & 1 & 1 & 2
\end{array} \text {. } \\
& \begin{array}{lllllllllllll}
0 & 0 & 1 & 1 & 0 & 0 & 1 & 1 & 1 & 1 & 0 & 0
\end{array}
\end{aligned}
$$

We call this common node $z$.

We now have 14 distinct nodes and it is easily checked that the graph obtained is $\operatorname{Inc}\left(\mathbb{P}_{2}\right)$. It is not possible to make any further $A_{7}$ extensions. Therefore $\Gamma=$ $\operatorname{Inc}\left(\mathbb{P}_{2}\right)$.

We now identify the group $G$. We do so by explicit enumeration of the root elements of $G$. As this has been described in detail in $[\mathbf{1}]$ we merely sketch the proof here here. We use the $Y_{333}$ reflection group along the relation of equation (2.5) and find that the 136 root elements represented by 


\begin{tabular}{|r|r|c|c|}
\hline$n$-gons & \# of nodes & graph & group \\
\hline 12 & 26 & $\operatorname{Inc}\left(\mathbb{P}_{3}\right)$ & $\mathbb{M} \imath 2$ \\
8 & 14 & $\operatorname{Inc}\left(\mathbb{P}_{2}\right)$ & $O_{8}^{-}(2): 2$ \\
6 & 10 & Petersen & $O_{6}^{-}(2): 2 \cong O_{5}(3): 2$ \\
6 & 8 & cube $=$ Inc (tetrahedron $)$ & $O_{5}(3) \times 2 \cong O_{6}^{-}(2) \times 2$ \\
\hline
\end{tabular}

TABLE 1. deflating $n$-gons in graphs to get groups

\begin{tabular}{|c|c|c|c|c|c|c|c|c|c|c|}
\hline 0 & + & - & & 0 & 0 & 0 & 1 & & 0 & 0 \\
\hline 0 & 0 & 0 & 0 & 0 & 0 & 0 & 1 & 1 & 0 & 0 \\
\hline $\begin{array}{ll}0 & 0\end{array}$ & 0 & 0 & & 0 & 0 & 0 & 1 & & 0 & 0 \\
\hline
\end{tabular}

form a conjugacy class of $G$. [The elements are obtained from the roots shown by permuting all four coordinates of each row and permuting the three rows. Parentheses show the number of distinct group elements obtained.]

These roots are then transformed by $G$ in exactly the same way that $O_{8}^{-}(2): 2$ permutes its root elements. [To see this view the 12 coordinates $a, \ldots, l$ modulo 2. The roots span a 10 dimensional subspace of $\mathbb{E}_{2}^{12}$. After applying the relations of equation (2.5) we get an 8 dimensional space $\mathbb{F}_{2}^{8}$. The bilinear form inherited from the quadratic form (equation (2.1)) has Witt defect 1.] It follows the resulting group is a central extension of $O_{8}^{-}(2): 2$. The multiplier of the simple group $O_{8}^{-}(2)$ is known to be 1 [2], therefore the extension is trivial and $G$ is $O_{8}^{-}(2): 2$.

This proves Theorem 2.3. We note again that Theorem 2.2 quickly follows since all the relations used in this proof are implied by the conditions of Theorem 2.2 and all relations of Theorem 2.2 hold in $O_{8}^{-}(2): 2$.

\section{Epilogue}

For reference we state the main theorems of $[\mathbf{5}]$.

THEOREM 3.1. If we deflate all free 12-gons of the Inc $\left(\mathbb{P}_{3}\right)$ Coxeter group we obtain the Bimonster $\mathbb{M}<2$.

THEOREM 3.2 (26 implies the Bimonster). Let $G$ be a group (of order greater than 2) generated by and satisfying the Coxeter relations of the $\mathbb{M}_{666}$ diagram. If after closure of the generating $\mathbb{M}_{666}$ diagram by adjoining $A_{11}$ extending nodes there are no more than 26 nodes, then $G$ is the Bimonster $\mathbb{M} 22$.

$\operatorname{Inc}\left(\mathbb{P}_{3}\right)$ is the (26 node) incidence graph of the projective plane of order 3 . The $\mathbb{M}_{666}$ diagram is the (16 node) $Y_{555}$ diagram.

The proofs of these theorems are similar those given in this paper. The principal complication is that the root enumeration would involve $|\mathbb{M}|$ (almost 10 ${ }^{54}$ ) root elements and therefore much less elementary methods $[\mathbf{8 , ~ 1 1 , 9 ]}$ are required to identify the group.

We also include a table (Table 1) from [5]. In this table we list the groups obtained by deflating all free $n$-gons of the Coxeter groups of certain graphs.

We are very interested in which other finite groups can be obtained as deflations of Coxeter groups. 


\section{References}

[1] J. H. Conway. From hyperbolic reflections to finite groups. In L. Finkelstein and W. M. Kantor, editors, Groups and Computation, number 11 in DIMACS Series in Discrete Mathematics and Theoretical Computer Science, pages 41-51, American Mathematical Society, 1993.

[2] J. H. Conway, R. T. Curtis, S. P. Norton, R.A. Parker, and R. A. Wilson. Atlas of Finite Groups. Oxford University Press, 1985.

[3] J. H. Conway, S. P. Norton, and L. H. Soicher. The Bimonster, the group $Y_{555}$, and the projective plane of order 3. In M. C. Tangara, editor, Computers in Algebra, number 111 in Lecture Notes in Pure and Applied Mathematics, pages 27-50. Marcel Dekker, 1988.

[4] J. H. Conway and A. D. Pritchard. Hyperbolic reflections for the Bimonster and $3 F i_{24}$. In Liebeck and Saxl [10], pages 24-45.

[5] J. H. Conway and C. S. Simons. 26 implies the Monster. Journal of Algebra, in press.

[6] J. H. Conway and C. S. Simons. Relations in $\mathbb{M}_{666}$. In R. T. Curtis and R. A. Wilson, editors, The Atlas 10 Years On, number 249 in London Mathematical Society Lecture Note Series, pages 27-38. Cambridge University Press, 1998.

[7] J. E. Humphreys. Reflection Groups and Coxeter Groups. Number 29 in Cambridge Studies in Advanced Mathematics. Cambridge University Press, 1990.

[8] A. A. Ivanov. A geometric characterization of the Monster. In Liebeck and Saxl [10], pages $46-62$.

[9] A. A. Ivanov. $Y$-groups via transitive extension. Journal of Algebra, 218:412-435, 1999.

[10] M. Liebeck and J. Saxl, editors. Groups, Combinatorics and Geometry, number 165 in London Mathematical Society Lecture Note Series. Cambridge University Press, 1992.

[11] S. P. Norton. Constructing the Monster. In Liebeck and Saxl [10], pages 63-76.

[12] C. S. Simons. Monster roots. In J. Ferrar and K. Harada, editors, The Monster and Lie Algebras, number 7 in Ohio State University Mathematical Research Institute Publications, pages 127-146. Walter de Gruyter, 1998.

Cicma, Defartment of Mathematics \& Statistics, Concordia University, MonTreal, Quebec H3G 1M8, Canada

Current address: Department of Mathematics, Rowan University, Glassboro, New Jersey, 08028, U.S.A.

E-mail address: simons@alumni.princeton .edu 